\title{
Isolation of trinucleotide microsatellite markers for Mystus nemurus
}

\begin{abstract}
Twelve single-locus trinucleotide microsatellite markers were developed to characterize the Asian river catfish, Mystus nemurus, an important food fish in Southeast Asia. They were obtained by using a rapid method, namely, the $5^{\prime}$ anchored PCR enrichment protocol. The specific primers were designed to flank the repeat sequences and these were subsequently used to characterize 90 unrelated fish from Malaysia. The number of alleles per locus ranged from $2(\mathrm{MnVj} 2-281)$ to $12(\mathrm{MnBp} 8-4-43 \mathrm{~b})$ while the levels of heterozygosity ranged from $0.0444(\mathrm{MnVj} 2-1-19)$ to $0.7458(\mathrm{MnVj2}-291)$.
\end{abstract}

Keyword: Trinucleotide microsatellite 